\title{
Study on Nanoscale Temperature Distribution for the Patterning of Self-Assembled Monolayers Using Near-Field Photothermal Desorption*
}

\author{
Yu YAMAMOTO**, Yoshihiro TAGUCHI*** and Yuji NAGASAKA*** \\ ** School of Integrated Design Engineering, Keio University, \\ 3-14-1 Hiyoshi, Kohoku-ku, Yokohama, Kanagawa, 223-8522, Japan \\ E-mail: yuyamamoto@naga.sd.keio.ac.jp \\ *** Department of System Design Engineering, Keio University, \\ 3-14-1 Hiyoshi, Kohoku-ku, Yokohama, Kanagawa, 223-8522, Japan
}

\begin{abstract}
We propose a novel patterning method for self-assembled monolayers (SAMs) using near-field photothermal desorption (NPTD). This paper reports the patterning principle and the study on optimum heating conditions. The proposed method utilizes the thermal desorption of constituent molecules of a SAM through the irradiation with near-field light, which can make noncontact and noncontaminating patterning of the SAMs at the nanoscale. In order to verify the validity of the proposed patterning method for SAMs, the preliminary patterning of a SAM by irradiating with a laser beam was performed. The results suggested that the constituent molecules were thermally desorbed and the subsequent modification of another kind of a SAM was successfully carried out. The numerical analysis of the temperature distribution after heating with near-field light was demonstrated by using the finite element method to investigate the heating condition of NPTD. The simulation results of laser heating well agreed with the preliminary experimental results, therefore, the applicability of the proposed analytical model was confirmed. Furthermore, the analytical results of the temperature distribution indicated that the local heating at the nanoscale with sufficient temperature increase for NPTD could be induced by the irradiation with near-field light generated by using an apertured fiber probe coated with Ag. As a result, the validity of the patterning principle and the optimal heating conditions were verified, and therefore, the possibility of the nanoscale patterning of SAMs using NPTD was confirmed.
\end{abstract}

Key words: Nanoscale Heat Transfer, Near Field Light, Photothermal Desorption, Self Assembled Monolayer, Temperature Distribution

\section{Introduction}

A self-assembled monolayer (SAM), which is an organic thin film formed by intermolecular forces and the interaction between the constituent molecules (typically thiolate: R-S-) and the substrate (typically $\mathrm{Au}$ ), has gained much attention and has been widely studied ${ }^{(1)-(4)}$, because of the great potential applications in various scientific and technological fields. For example, SAMs are often used to change the surface adhesion, corrosion, wettability, conductivity and reactivity of other molecules. Feng et al. studied the corrosion protection imparted by a SAM formed on a $\mathrm{Cu}$ substrate ${ }^{(5)}$. It was revealed that it showed much better corrosion resistance than other inhibitors, and a method of forming

DOI: $10.1299 /$ jtst.6.436]

Copyright $\mathbb{C} 2011$ by JSME 
such monolayers that improved corrosion protection significantly was established. Boulas et al. improved energy barriers of an insulator used in an electronic device by forming a SAM on a Si substrate ${ }^{(6)}$. Such suppression of charge carrier tunneling in electronic devices was achieved because of the high insulating capacity of the SAM. Moreover, in recent years, patterned SAMs have been developed in order to address wide-ranging applications in nano/micro devices. For example, nano-patterned SAMs can be applied to surface-enhanced Raman scattering (SERS) active sites ${ }^{(7)}$, uniformly sized nanocatalysts ${ }^{(8)}$, and biomolecular sensors $^{(9)}$.

In terms of the above aspects, nanoscale patterning is necessary for such applications of SAMs, and various patterning methods have been invented. Electron beam lithography can decompose a SAM at the nanoscale ${ }^{(10)-(13)}$, however, irradiation with an electron beam induces the cleavage of carbon chains and the formation of new undesirable bonds, resulting in an organic residue at the patterning site. Although micro contact printing has the advantages of high throughput and large-area patterning ${ }^{(14)-(17)}$, deformation of the mold induces defects in the film and restricts the pattern capability. Some researchers have studied the patterning methods using the laser excitation. Klingebiel et al. have investigated the photothermal laser processing of alkylsiloxane monolayers formed on a $\mathrm{SiO}_{2}$ substrate $^{(18)}$. However, the remaining silanol at the irradiated area may cause the restriction of the application possibility, and the patterning scale was restricted to micrometer scale due to the diffraction limit of light. As another example using laser excitation, Chang et al. have made SAMs patterns using femtosecond laser and SAMs interaction ${ }^{(19)}$. They utilized photochemical reaction between the Au substrate and alkanethiolate for making the SAMs patterning. However, there is a contamination issue caused by the decomposition of the carbon chains and the formation of new undesirable organics at the irradiated area other than the break of the bond between the Au substrate and sulfur atoms. Also, the mechanism of this photochemical reaction has been the subject of some debate. Thus, general patterning methods are restricted to specific applications in nanotechnology.

From above aspects, we have proposed a novel patterning method for SAMs, which uses near-field photothermal desorption (NPTD), enabling noncontact, nondestructive and noncontaminating patterning of the SAMs at the nanoscale. Therefore, this method can be applied in the pursuit of various objectives in nanotechnology, especially techniques that need extremely clean and well-ordered surface such as soft-landing method ${ }^{(20)}$. For the near-field photothermal desorption of SAMs, the accurate control of heating conditions such as optical intensity, material of near-field fiber probe and substrate are quite important. This paper reports the principle of the nanoscale patterning of SAMs, and the optimal heating condition of NPTD that is numerically analyzed by using the FEM.

\section{Patterning Principle}

In our method, near-field light and thermal desorption of constituent molecules (thiolates in our case) are utilized to carry out the patterning of SAMs at the nanoscale. Figure 1 shows a schematic image of the principle of NPTD. The sample surface is locally heated using near-field light, and then bound molecules desorb thermally due to the break of the bond between the substrate and thiolates. The desorption of thiolate is induced at high temperatures $\left(400 \sim 500 \mathrm{~K}^{(21)(22)}\right)$. This process does not need pretreatment such as coating of a photoresist and can remove the thiolate thermally from the substrate without the formation of organic residue because the desorption is resulted from the break of the bond between the substrate and thiolate, and thereby thiolates at the irradiated area are removed, resulting in no remaining organic at the irradiated area. Therefore, a direct and nondestructive patterning can be carried out. Moreover, because the local heating is induced through the irradiation with near-field light that is localized in the proximity of a small 


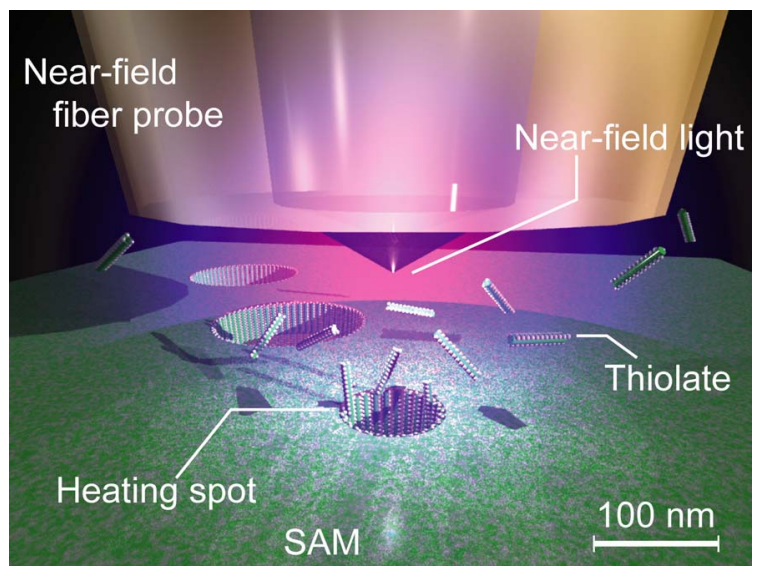

Fig. 1 Illustration of the principle of the proposed patterning method (NPTD)

aperture, whose diameter is smaller than the wavelength of the incident light, our method can pattern SAMs at the nanoscale beyond the diffraction limit of light. In order to generate near-field light, a near-field fiber probe coated with metal is used. Irradiation with the near-field light for the heating of the sample surface can be performed by keeping the near-field fiber probe in the proximity of the sample surface. The size of the desorption area can be controlled by adjusting the surface temperature, which depends on both the size of the near-field light and its energy density.

\section{Experimental Study on Photothermal Desorption of SAM}

\subsection{Overview of the preliminary experiment}

In order to verify the validity of the proposed patterning method for a SAM using photothermal desorption, preliminary patterning of an octadecanethiol (ODT: $\left.\mathrm{CH}_{3}\left(\mathrm{CH}_{2}\right)_{17} \mathrm{SH}\right) \mathrm{SAM}$ using irradiation with a laser beam was demonstrated. The procedure of the preliminary patterning is summarized in the following three steps:

(1) Initial formation of ODT-SAM

A $10 \times 10 \mathrm{~mm}^{2}$ triple-layered substrate, $\mathrm{Au}$ (thickness: $100 \mathrm{~nm}$ ) $/ \mathrm{Ti} / \mathrm{SiO}_{2}$, was immersed in piranha solution $\left(3: 1\right.$ concentrated $\left.\mathrm{H}_{2} \mathrm{SO}_{4} / \mathrm{H}_{2} \mathrm{O}_{2}\right)$ for 20 min to remove organic contaminants from the surface ${ }^{(1)(4)}$. By dipping the substrate into a $1.0 \mathrm{mM}$ ethanolic solution of ODT at ambient temperature for $24 \mathrm{~h}$, ODT-SAM was formed uniformly on the sample surface. After the formation of ODT-SAM, the sample was carefully rinsed with ethanol. Figure 2 shows a schematic image of ODT-SAM formed on a triple-layered substrate. Through this modifying process, the thiolate can chemisorb to the Au surface, and this chemisorption and the intermolecular force can create a uniform and well-ordered ODT-SAM on the Au surface as shown in Fig. 2. The surface characterization of the formed ODT-SAM is performed by infrared reflection absorption spectroscopy (IRAS).

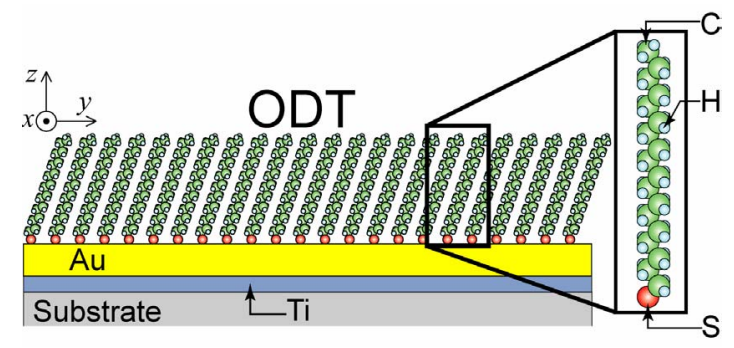

Fig. 2 Schematic of ODT-SAM formed on an Au surface 
(2) Irradiation with laser beam

After the formation of the ODT-SAM, the sample was irradiated with a laser beam to induce local desorption of ODT thermally. Figure 3 shows the schematic of the optical setup. A laser diode (wavelength: $405 \mathrm{~nm}$ ) and a He-Ne laser (wavelength: $633 \mathrm{~nm}$ ) were employed and the two laser beams were aligned on the same optical path by using a dichroic mirror (DM). The laser diode was used to heat up the sample, and the He-Ne laser was used to align the irradiation position for the heating laser diode. The He-Ne laser was utilized for the pre-alignment of the heating position because alignment with the laser diode would induce damage in the sample surface $(\mathrm{Au})$ through absorption of the laser beam. The laser diode was guided by using the pre-aligned He-Ne laser beam. The intensity of the heating laser could be controlled with a half wavelength plate $(\lambda / 2)$ and a polarized beam splitter (PBS). Also, back-reflection could be eliminated using a combination of the same optics and a quarter wavelength plate $(\lambda / 4)$. The diameter of the laser beam on the sample was controlled with a beam expander (BE) and a condenser lens.

(3) Modification of another kind of SAM

After irradiation with the laser beam, the sample was immersed in a $1.0 \mathrm{mM}$ ethanolic solution of 11-amino-1-undecanethiol (AUT: $\mathrm{NH}_{2}\left(\mathrm{CH}_{2}\right)_{11} \mathrm{SH}$ ) at ambient temperature. This process induced the modification of the AUT in the irradiated area, and then the patterning of the SAM was performed.

To evaluate the patterning after irradiation with the laser beam and modification of the AUT, a scanning electron microscope (SEM) was used. Since the contrast difference in a SEM image is attributed to the differences between the emission capabilities of secondary electrons in the surface molecules ${ }^{(23)}$, the nanoscale patterning of SAMs can be observed, and the patterning capability can be evaluated.

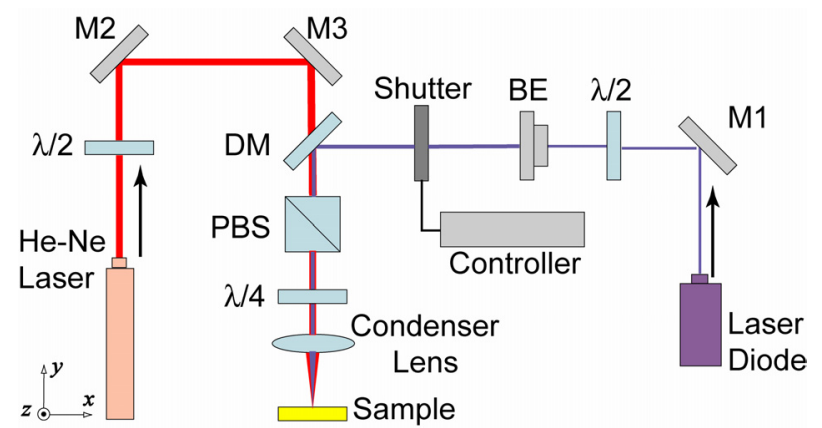

Fig. 3 Schematic of the optical setup for irradiation with a laser beam of the sample

\subsection{Experimental Results and Discussion}

\subsubsection{Characterization of ODT-SAM}

Figure 4 shows the $\mathrm{C}-\mathrm{H}$ stretching region $\left(2700 \sim 3100 \mathrm{~cm}^{-1}\right)$ of the measured IRAS spectra for the formed ODT-SAM. The spectrum peaks corresponding to antisymmetric and symmetric stretching modes of the terminal methyl group and methylene group of carbon chains were measured as shown in Table 1. These data were in good accordance with previously reported IRAS spectra ${ }^{(24)}$. Therefore ODT-SAM was successfully formed on Au by the above process.

\subsubsection{Evaluation of the patterning of SAM}

Laser irradiation was performed for two patterns: a continuous linear pattern and a periodic pattern. Irradiation for the continuous pattern was carried out by changing the laser power from $5 \mathrm{~mW}$ to $45 \mathrm{~mW}$ at a Gaussian radius of $4 \mu \mathrm{m}$ and a scan speed of $200 \mu \mathrm{m} / \mathrm{s}$. Figure 5 shows a SEM image of the irradiated area. The gray region indicates ODT, which 


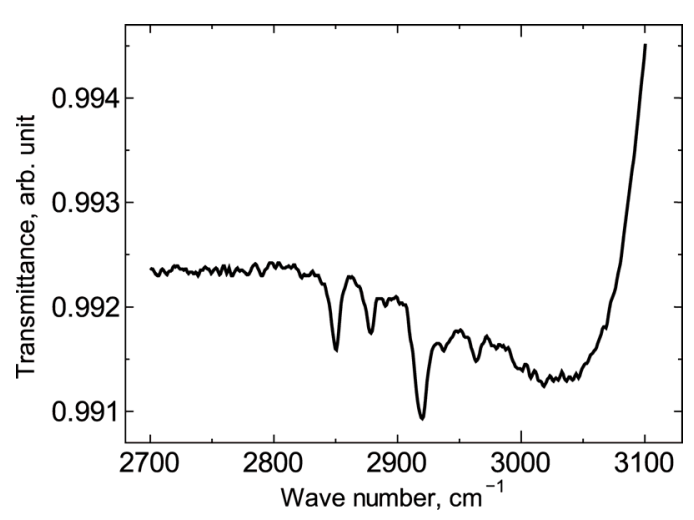

Fig. 4 IRAS spectra of the formed ODT-SAM

Table 1 Measured peak wave number and its assignments

\begin{tabular}{ccc}
\hline \hline Measured wave number, $\mathrm{cm}^{-1}$ & Assignment & Reference wave number, $\mathrm{cm}^{-1}$ \\
\hline \hline 2851 & C-H symmetric stretching mode $\left(\mathrm{CH}_{2}\right)$ & 2850 \\
\hline 2878 & C-H symmetric stretching mode $\left(\mathrm{CH}_{3}\right)$ & 2878 \\
\hline 2920 & C-H antisymmetric stretching mode $\left(\mathrm{CH}_{2}\right)$ & 2917 \\
\hline 2936 & C-H symmetric stretching mode $\left(\mathrm{CH}_{3}\right)$ & 2937 \\
\hline 2963 & C-H antisymmetric stretching mode $\left(\mathrm{CH}_{3}\right)$ & 2960 \\
\hline \hline
\end{tabular}

is highly able to emit secondary electrons, and the darker linear regions indicate AUT, which is less able to emit secondary electrons. The darker linear regions suggest that ODT was thermally desorbed by irradiation with the laser beam, and AUT was successfully modified in the irradiated area, resulted in the noncontaminating patterning. It was also observed that the contrast and the width of the lines became clearer and wider as the sample was irradiated with higher laser beam intensities in the case of the laser power of over 45 $\mathrm{mW}$ at a Gaussian radius of $4 \mu \mathrm{m}$. However, because the surface temperature didn't reach the temperature level of desorption at the laser power of less than $23 \mathrm{~mW}$, no line features could be determined.

In order to quantify the experimental SEM results, the SEM image was postprocessed to extract the pixel intensity values in 256 shades of gray corresponding to the degree of desorption of ODT using Matlab. The contrast difference in a SEM image is attributed to the differences between the emission capabilities of secondary electrons in the surface molecules, and the emission capability of secondary electrons depends on the surface molecule. Therefore the contrast difference in a SEM image is proportional to the fraction of AUT that was modified in the irradiated area, and the degree of the desorption can be evaluated by the pixel intensity value analysis. The pixel intensities for each scan across the line features were recorded and used to produce pixel intensity profiles. To reduce noise components, the resulting pixel intensity profiles were averaged in the x-direction. Figure 6 shows the pixel intensity values obtained from the SEM image of the irradiated area, and the approximate desorption area is evaluated by the full width at half-maximum (FWHM). The peak intensity became larger and the desorption width became wider as the sample was irradiated with higher laser beam intensities. The pixel intensity in the case of $45 \mathrm{~mW}$ was almost twice than that in the case of $23 \mathrm{~mW}$ at a Gaussian radius of $4 \mu \mathrm{m}$, and FWHM of the desorption width was about $5 \mu \mathrm{m}$ at the laser power of $23 \mathrm{~mW}$. Consequently, the laser power and the Gaussian radius, which is corresponding to the energy density of the laser beam, are important factors in the desorption of thiolate, and the desorption width can be controlled by changing the laser power. 


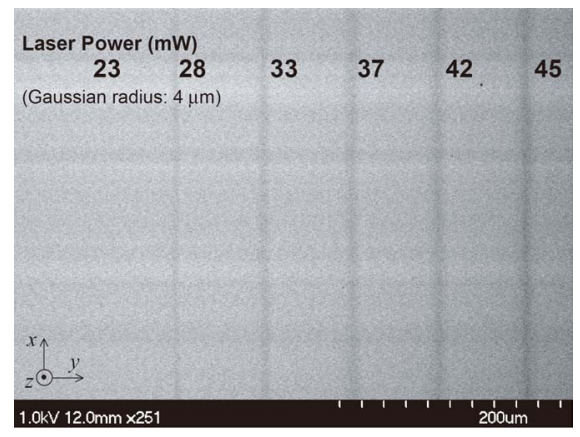

Fig. 5 SEM image of the irradiated area

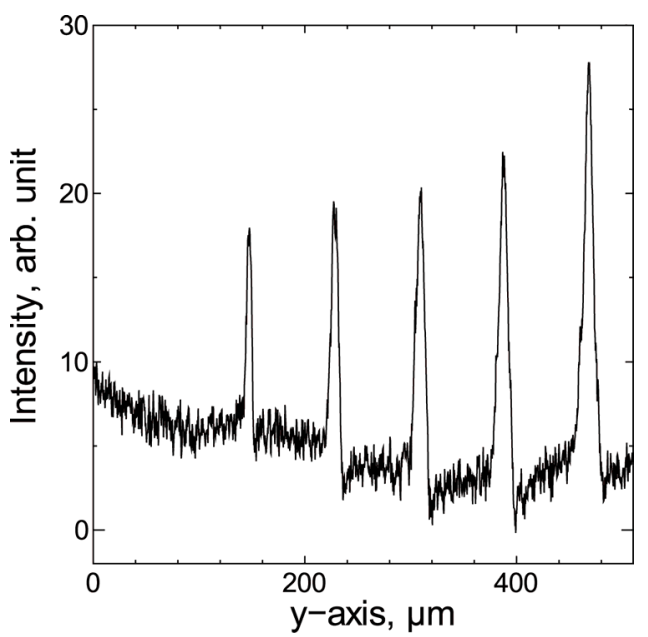

Fig. 6 Pixel intensity values obtained from the SEM image(Fig. 5) of the irradiated area

Next, the periodic irradiation was carried out at the laser power of $45 \mathrm{~mW}$, and a Gaussian radius of $4 \mu \mathrm{m}$ with the irradiation time set as $1 \mathrm{~s}$. The shutter shown in Fig. 3 was used for this periodic irradiation. Figure 7(a) shows a SEM image of the irradiated area. The gray region and the darker circular regions indicate ODT and AUT, respectively, as shown in Fig. 7(b). The contrast difference suggests that ODT was thermally desorbed and AUT was successfully modified in the case of periodic irradiation as well as in the case of the continuous linear irradiation. The periodic irradiation was also investigated using pixel intensity values as noted above. Figure 8 shows the pixel intensity values obtained from the SEM image shown in Fig. 7(a). The intensity changed drastically at the edge of the

(a)

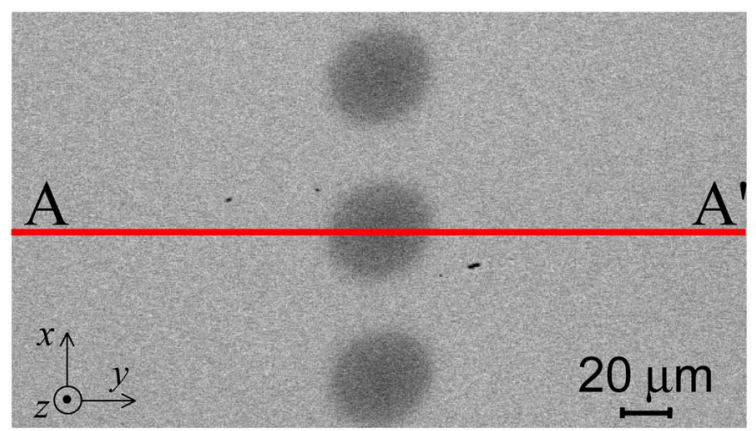

(b)

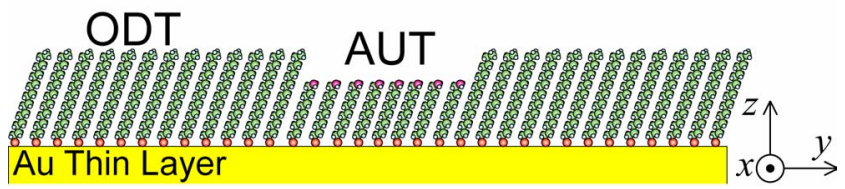

Fig. 7 (a) SEM image of the irradiated area

(b) Schematic of the cross-section along A-A' in (a) 


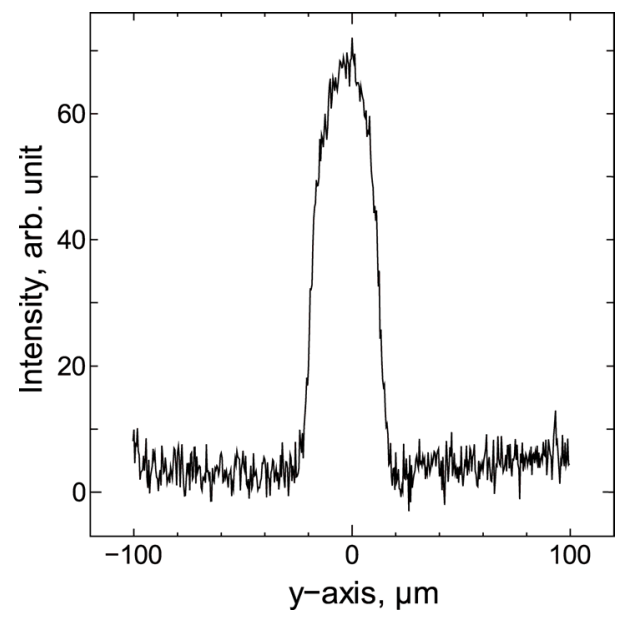

Fig. 8 Pixel intensity values obtained from the SEM image(Fig. 7(a)) of the irradiated area

desorption area. Therefore, ODT and AUT existed separately at the sample surface. The full width at half-maximum of the desorption area was about $30 \mu \mathrm{m}$. The enlargement of the desorption area compared with the heated area (a Gaussian radius: $4 \mu \mathrm{m}$ ) was due to the heat conduction.

As described above, the formation and the thermal desorption of ODT-SAM were successfully performed. Furthermore, it was demonstrated that the patterning area could be controlled by changing the laser power appropriately.

\section{Numerical Analysis by using FEM}

In order to investigate the optimal heating condition of NPTD, the numerical analysis of the temperature distribution after heating with near-field light was carried out by using the FEM. Table 2 shows the material properties used in this analysis.

Table 2 Material properties ${ }^{(25)(26)}$

\begin{tabular}{ccccc}
\hline \hline Material & Density & Specific heat & $\begin{array}{c}\text { Thermal } \\
\text { conductivity } \\
\mathrm{W} /(\mathrm{m} \cdot \mathrm{K})\end{array}$ & $\begin{array}{c}\text { Absorptance } \\
(\text { at } 445 \mathrm{~nm})\end{array}$ \\
\hline $\mathrm{kg} / \mathrm{m}^{3}$ & $\mathrm{~J} /(\mathrm{kg} \cdot \mathrm{K})$ & $3.17 \times 10^{2}$ & 0.61 \\
\hline $\mathrm{Ag}$ & $1.93 \times 10^{4}$ & $1.29 \times 10^{2}$ & $4.29 \times 10^{2}$ & 0.08 \\
$\mathrm{Si}$ & $1.05 \times 10^{4}$ & $2.35 \times 10^{2}$ & $1.45 \times 10^{2}$ & - \\
\hline $\mathrm{SiO}_{2}$ & $2.33 \times 10^{3}$ & $7.02 \times 10^{2}$ & 1.40 & - \\
\hline \hline
\end{tabular}

\subsection{Analysis in case of microscale heating}

As a stage prior to the nanoscale heating by the near-field light, the analytical model of

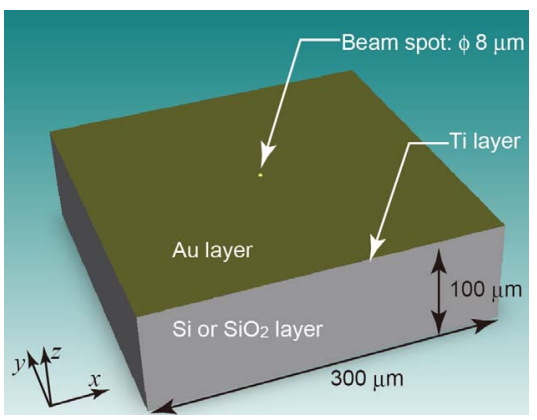

Fig. 9 Three dimensional analytical model of a substrate 
(a)

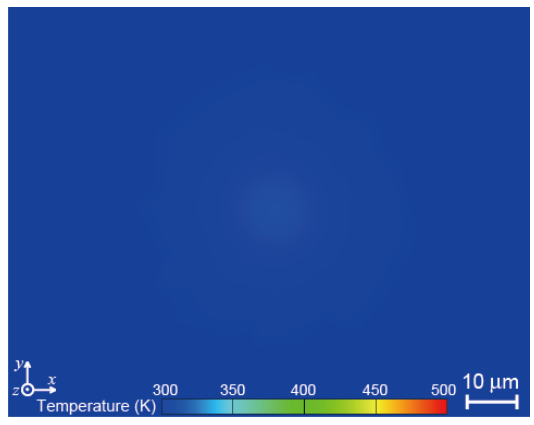

(b)

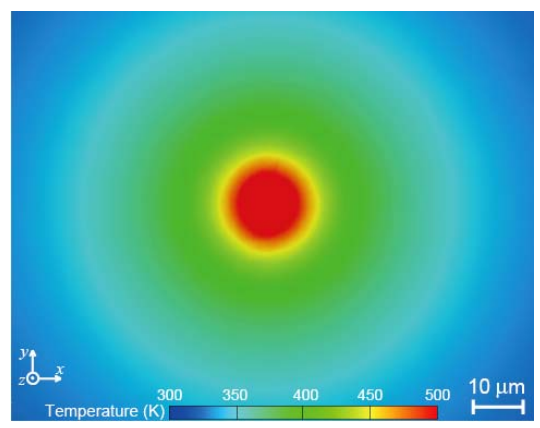

Fig. 10 Analysis results of the temperature distribution around the heated area (a) in the case of $\mathrm{Si}$ and (b) in the case of $\mathrm{SiO}_{2}$ as the material of the bottom layer

the laser irradiation was created and analyzed to verify the validity of the patterning principle of photothermal desorption which utilized the heating of the substrate by the irradiation of light. First, the influence of the material of the substrate was investigated. As the sample of the analytical model, a triple-layer structure $\left(\mathrm{Au} / \mathrm{Ti} / \mathrm{Si}\right.$ or $\left.\mathrm{SiO}_{2}\right)$ was constructed as shown in Fig. 9. The material of the bottom layer of the model was $\mathrm{Si}$ and $\mathrm{SiO}_{2}$, which were commonly-used for the substrate of the formation of Au thin film. The thicknesses of each layer were $100 \mathrm{~nm}, 50 \mathrm{~nm}, 100 \mu \mathrm{m}$ respectively, and the horizontal size of the model was $300 \mu \mathrm{m}$. The diameter of the heated area was $8 \mu \mathrm{m}$, and the laser power was $45 \mathrm{~mW}$. The optical absorptance of Au was 0.61 at the wavelength of $445 \mathrm{~nm}$. Figure 10 is the analysis result for the temperature distribution around the heated area in the case of Si bottom layer (Fig. 10(a)) and $\mathrm{SiO}_{2}$ bottom layer (Fig. 10(b)). In the case of Si, there was little temperature increase at the sample surface compared with $\mathrm{SiO}_{2}$. Because of the high conductivity of Si bottom layer, the heat conduction toward the Si bottom layer was dominant, thus it was difficult to desorb thiolates from the substrate of Si. On the other hand, in the case of $\mathrm{SiO}_{2}$, the concentric temperature distribution was generated and the temperature of the center reached $600 \mathrm{~K}$, which was above to the temperature of the desorption of thiolates. Therefore, it was suggested that the thermal desorption of thiolates could be induced by using the substrate of $\mathrm{SiO}_{2}$.

Figure 11 shows the numerical result of temperature distribution around the heated area shown in Fig. 10(b). The width of the desorption area was estimated to be about $20 \sim$ $35 \mu \mathrm{m}$ when the desorption temperature of ODT was $400 \sim 500 \mathrm{~K}$. Compared with the diameter of the heated area $(8 \mu \mathrm{m})$, the diameter of the desorption area was larger because of the heat conduction of the sample. Compared with the experimental result shown in Fig. 8 , this analysis result was in good agreement with the experimental result. Therefore, the photothermal desorption width can be estimated by the proposed analytical model. 


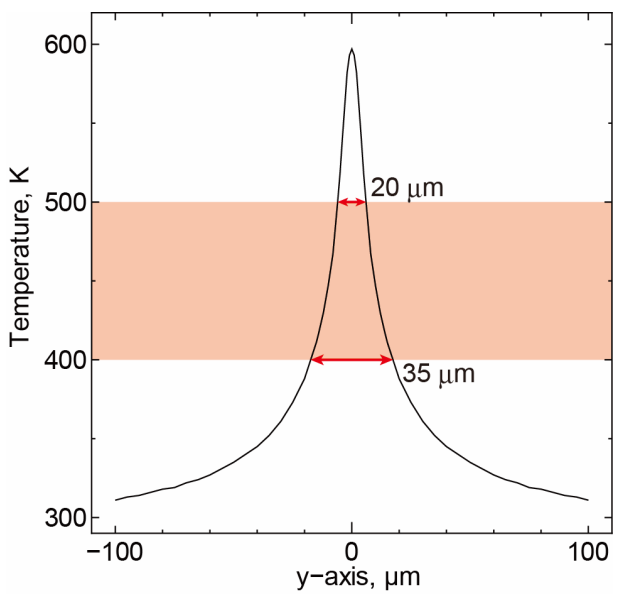

Fig. 11 Simulation result for the temperature distribution

\subsection{Analysis in case of nanoscale heating}

Finally, numerical analysis of the temperature distribution after heating with near-field light was carried out. The near-field fiber probe is utilized to generate and irradiate with the near-field light. Since the near-field light is generated in a proximity region of the fiber probe, it is necessary to consider the heat conduction between the near-field fiber probe and the sample. Also, the heat generation at the fiber probe due to the light absorption of the near-field fiber probe should be considered. Figure 12 shows a cross-sectional image of the

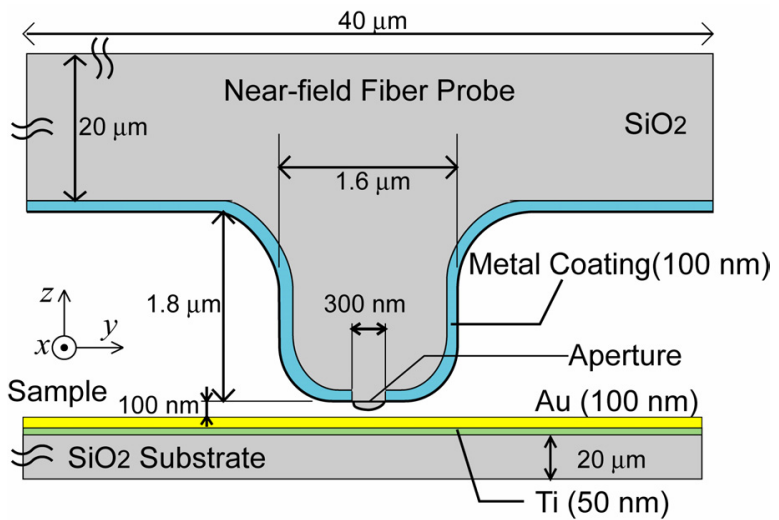

Fig. 12 Cross-sectional schematic of the analytical model of the near-field fiber probe (upper part) and the sample (lower part)

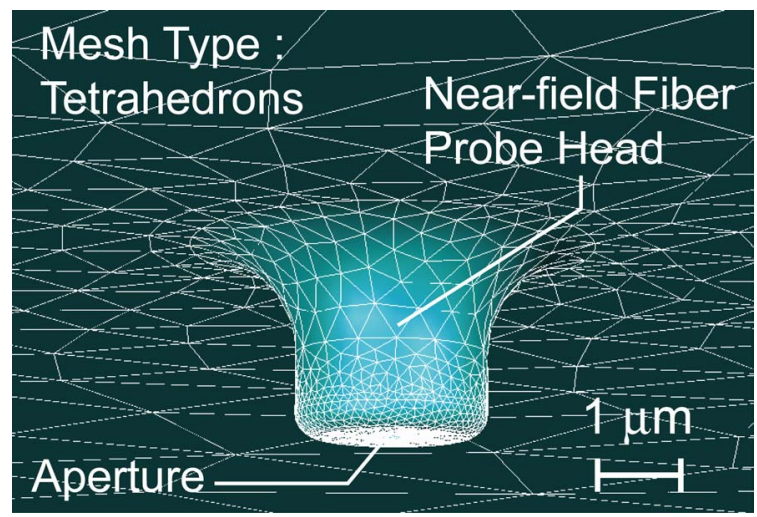

Fig. 13 Three-dimensional analytical model based on the two-dimensional layout shown in Fig. 12 
analytical model. The upper part is a near-field fiber probe. A thin metal coating was sputtered onto $\mathrm{a} \mathrm{SiO}_{2}$ fiber probe head sharpened by wet etching to generate near-field light. As the coating metal, Ag or Au was used. And, an aperture of $300 \mathrm{~nm}$ in diameter was created at the tip of the probe. As the sample of the analytical model, a triple-layer structure $\left(\mathrm{Au} / \mathrm{Ti} / \mathrm{SiO}_{2}\right)$ was constructed. Figure 13 shows a three-dimensional analytical model of a near-field fiber probe head with an aperture at the tip of the probe head. In this analysis, not only the absorption of the near-field light at the sample surface but also that of the excitation laser beam at the probe head coated with Au or Ag was considered. The material values of these two metals used in this analysis are shown in Table 2. A tetrahedron mesh type was used, and a finer mesh was generated at the probe head and the heated area, which allows more precise analysis. The wavelength of the excitation laser beam was $445 \mathrm{~nm}$ and the excited laser power was about $50 \mathrm{~mW}$. The diameter of the heated area was $300 \mathrm{~nm}$. Although a near-field fiber probe is known to have the low energy transmittance in general, a double-tapered near-field fiber probe was utilized in our method. This probe is fabricated by the chemical etching using two different concentration of buffered HF solution. By using this probe, high coupling efficiency (about $1 \sim 10 \%$ ) can be achieved, and thereby the input energy is transferred to the sample effectively. The analysis was conducted under steady state condition.

Figure 14 shows the analysis results for the temperature distribution after heating in the case of Au (Fig. 14(a)) and Ag (Fig. 14(b)) as the coating metal. In Fig. 14(a), the temperature of the probe head was extremely high due to the absorption of the excitation laser beam by the Au coating. This absorption and following heat conduction along the $\mathrm{Au}$ coating were a large loss of energy of the excitation laser beam, and were thought to have become the heat source for the sample. On the other hand, in the case of Ag as the coating metal, a high temperature was only generated at the sample surface around the irradiated area, because the Ag coating with a low optical absorptance prevented an increase in

(a)

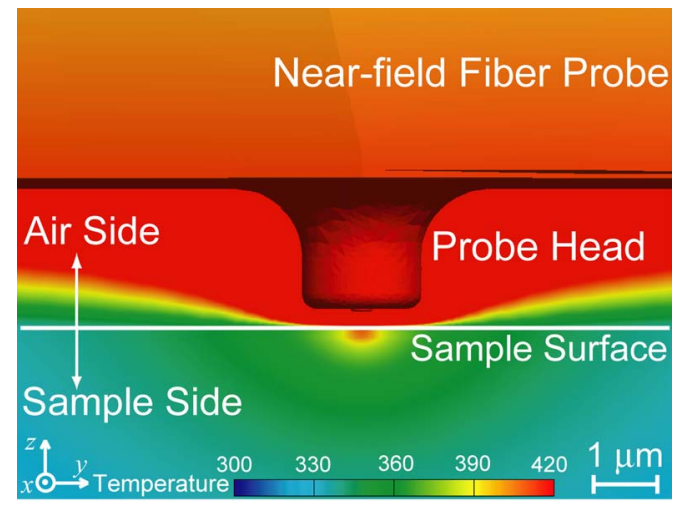

(b)

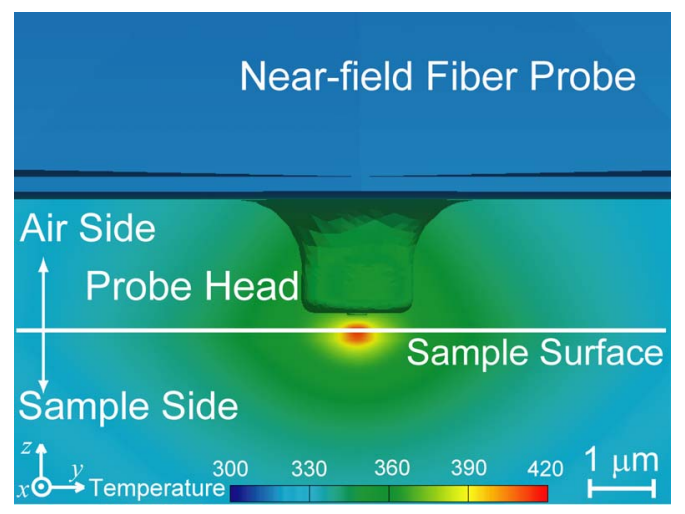

Fig. 14 Analysis results for the temperature distribution (a) in the case of $\mathrm{Au}$ and (b) in the case of Ag as the coating metal 
temperature at the probe head and led to local heating at the sample surface at the nanoscale without any other heat source. Figure 15 shows the analysis result of the distribution of the heat flux magnitude after heating (coating material of Ag). The sample surface was locally heated and the high heat flux was generated at the nanoscale area resulting from the irradiation with the near-field light. On the other hand, the heat flux from the probe head due to the absorption of the excited light was negligibly small. Figure 16 shows the temperature distribution around the heated area when the excited laser power is about 50 $\mathrm{mW}$ (shown in Fig. 14(b)). In this case, when the threshold of the desorption temperature was estimated to be $400 \mathrm{~K}$, the desorption width was around $300 \mathrm{~nm}$, which was the same as the irradiated area (and the diameter of the aperture). Consequently, the desorption width can be controlled at the nanoscale by changing the energy density of the near-field light generated by using a near-field fiber probe coated with Ag.

In this paper, we showed the advantage of $\mathrm{Ag}$ as the coating metal of a near-field fiber probe for NPTD. However it is known that the silver is affected by oxidation. In this case, the hybrid coating probe that is coated with another metal on Ag film is required to prevent the oxidation of Ag coating.

\section{Conclusion}

A novel patterning method for SAMs using NPTD enabling noncontact, nondestructive and noncontaminating patterning of the SAMs was proposed. The preliminary experimental results indicated that the desorption of ODT through irradiation with a laser beam and the modification of AUT were successfully performed. In the numerical analysis, the applicability of the estimation of photothermal desorption width was confirmed. Moreover,

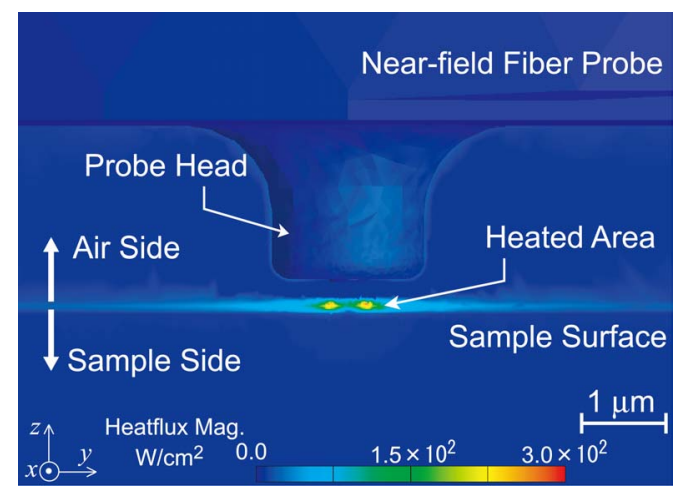

Fig. 15 Analysis result for the distribution of the heat flux magnitude

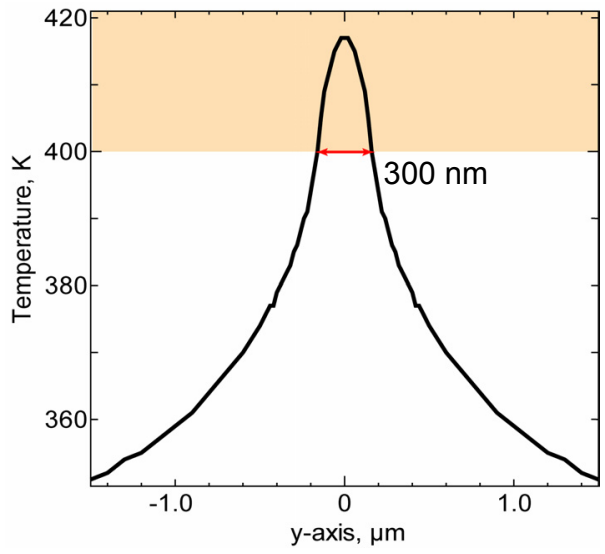

Fig. 16 Analysis results for the temperature distribution around the heated area in the case of Ag as the coating metal 
the analytical results indicated that the sample surface was locally heated at the nanoscale by near-field light generated by using the near-field fiber probe coated with Ag. As a result, the possibility of the nanoscale patterning of the SAMs using NPTD was confirmed.

\section{Acknowledgements}

This research was partially supported by the Japan Society for the Promotion of Science (JSPS), a Grant-in-Aid for Scientific Research (S, No. 19106004), and the Ministry of Education, Culture, Sports, Science and Technology (MEXT), Grant-in-Aid for young scientist (A, No. 23686036).

\section{References}

(1) Love, J. C., Estroff, L. A., Kriebel, J. K., Nuzzo, R. G. and Whitesides, G. M., "Self-Assembled Monolayers of Thiolates on Metals as a Form of Nanotechnology" Chemical Reviews, Vol. 105, No. 4 (2005), pp. 1103-1170.

(2) Dubois, L. H. and Nuzzo, R. G., "Synthesis, Structure, and Properties of Model Organic Surfaces", Annual Review of Physical Chemistry, Vol. 43 (1992) pp. 437-463.

(3) Ulman, A., "Formation and Structure of Self-Assembled Monolayers", Chemical Reviews, Vol. 96, No. 4 (1996), pp. 1533-1554.

(4) Schreiber, F., "Structure and Growth of Self-Assembling Monolayers", Progress in Surface Science, Vol. 65 (2000), No. 5-8, pp. 151-256.

(5) Feng, Y., Teo, W., Siow, K., Gao, Z., Tan, K. and Hsieh, A., "Corrosion Protection of Copper by a Self-Assembled Monolayer of Alkanethiol", Journal of The Electrochemical Society, Vol. 144, No. 1 (1997), pp. 55-64.

(6) Boulas, C., Davidovits, J. V., Rondelez, F. and Vuillaume, D., "Suppression of Charge Carrier Tunneling through Organic Self-Assembled Monolayers", Physical Review Letters, Vol. 76, No. 25 (1996), pp. 4797-4800.

(7) Song, W., Li, W., Cheng, Y., Jia, H., Zhao, G., Zhou, Y., Yang, B., Xu, W., Tian, W. and Zhao, B., "Surface Enhanced Raman Scattering from a Hierarchical Substrate of Micro/Nanostructured Silver", Journal of Raman Spectroscopy, Vol. 37, No. 7 (2006), pp. 755-761.

(8) Lu, J., Kopley, T., Dutton, D., Liu, J., Qian, C., Son, H., Dresselhaus, M. and Kong J., "Generating Suspended Single-Walled Carbon Nanotubes Across a Large Surface Area via Patterning Self-Assembled Catalyst-Containing Block Copolymer Thin Films", The Journal of Physical Chemistry B, Vol. 110, No. 22 (2006), pp. 10585-10589.

(9) Baldrich, E., Laczka, O., Campo, F. J. D. and Munoz, F. X., "Self-Assembled Monolayers as a Base for Immunofunctionalisation: Unequal Performance for Protein and Bacteria Detection", Analytical and Bioanalytical Chemistry, Vol. 390, No. 6 (2008), pp. 1557-1562.

(10) Zharnikov, M., Geyer, W., Goelzhaeuser, A., Frey, S. and Grunze, M., "Modification of Alkanethiolate Monolayers on Au-Substrate by Low Energy Electron Irradiation: Alkyl Chains and the S/Au Interface", Physical Chemistry Chemical Physics, Vol. 1, No. 13 (1999), pp. 3163-3171.

(11) Calvert, J.M., "Lithographic Patterning of Self-Assembled Films", Journal of Vacuum Science \& Technology B, Vol. 11, No. 6 (1993), pp. 2155-2163.

(12) Sondag-Huethorst, J. A. M., van Helleputte, H. R. J. and Fokkink, L. G. J., "Generation of Electrochemically Deposited Metal Patterns by means of Electron Beam (Nano)lithography of Self-Assembled Monolayer Resists", Applied Physics Letters, 
Vol. 64, No. 3 (1994), pp. 285-287.

(13) Parikh, A. N., Seshadri, K. and Allara, D. L., "Sub-10 nm Lithography with Self-Assembled Monolayers", Applied Physics Letters, Vol. 68, No. 11 (1996), pp. 1504-1506.

(14) Kumar, A., Biebuyck, H. A. and Whitesides, G. M., "Patterning Self-Assembled Monolayers - Applications in Materials Science", Langmuir, Vol. 10, No. 5 (1994), pp. 1498-1511.

(15) Delamarche, E., Schmid, H., Michel, B. and Biebuyck, H., "Stability of Molded Polymethylsiloxane Microstructures", Advanced Materials, Vol. 9, No. 9 (1997), pp. 741-746.

(16) Larsen, N. B., Biebuyck, H., Delamarche, E. and Michel, B., "Order in Microcontact Printed Self-Assembled Monolayers", Journal of The American Chemical Society, Vol. 119, No. 13 (1997), pp. 3017-3026.

(17) Farm, E., Kemell, M., Ritala, M. and Leskela, M., "Selective-Area Atomic Layer Deposition with Microcontact Printied Self-Assembled Octadecyltrichlorosilane Monolayers as Mask Layers”, Thin Solid Films, Vol. 517, No. 2 (2008), pp. 972-975.

(18) Klingebiel, B., Schroter, A., Franzka, S. and Hartmann, N., "Photothermally Induced Bromination and Decomposition of Alkylsiloxane Monolayers on Surface-Oxidized Silicon Substrates", Journal of Vacuum Science \& Technology A, Vol. 28, No. 4 (2010), pp. 834-837.

(19) Chang, W., Choi, M., Kim, J., Cho, S. and Whang, K., "Sub-Micron Scale Patterning Using Femtosecond Laser and Self-Assembled Monolayers Interaction”, Applied Surface Science, Vol. 240, No. 1-4 (2005), pp. 296-304.

(20) Mitsui, M., Nagaoka, S., Matsumoto, T. and Nakajima, A., "Soft-Landing Isolation of Vanadium-Benzene Sandwich Clusters on a Room-Temperature Substrate Using n-Alkanethiolate Self-Assembled Monolayer Matrixes", The Journal of Physical Chemistry B, Vol. 110, No. 7 (2006), pp. 2968-2971.

(21) Nishida, N., Hara, M., Sasabe, H. and Knoll, W., "Thermal Desorption Spectroscopy of Alkanethiol Self-Assembled Monolayer on Au(111)", Japanese Journal of Applied Physics, Vol. 35, No. 1 (1996), pp. 5866-5872.

(22) Delamarche, E., Michel, B., Kang, H. and Gerber, C., "Thermal Stability of Self-Assembled Monolayers", Langmuir, Vol. 10, No. 11 (1994), pp. 4103-4108.

(23) Lopez, G. P., Biebuyck, H. A. and Whitesides, G. M., "Scanning Electron Microscopy Can Form Images of Patterns in Self-Assembled Monolayers", Langmuir, Vol. 9, No. 6 (1993), pp. 1513-1516.

(24) Porter, M. D., Bright, T. B., Allara, D. L. and Chidsey, C. E. D., "Spontaneously Organized Molecular Assemblies. 4. Structural Characterization of n-Alkyl Thiol Monolayers on Gold by Optical Ellipsometry, Infrared Spectroscopy, and Electrochemistry", Journal of the American Chemical Society, Vol. 109, No. 12 (1987), pp. 3559-3568.

(25) Palik, E. D. ed., Handbook of optical constants of solids, (1995), p. 294, 356, Academic Press.

(26) Japan society of Thermophysical Properties ed., Thermophysical Properties Handbook, (2008), pp. 20-22, 300, Yokendo. 